\title{
Robot-assisted partial nephrectomy and nephrolithotomy for multiple lower pole calculi in an atrophic kidney
}

\begin{abstract}
Open renal stone surgery has been largely replaced by retrograde intrarenal surgery (RIRS), percutaneous nephrolithotomy (PCNL) and extracorporeal shockwave lithotripsy (ESWL). However, when these methods fail to remove a stone or there are certain contraindications, such as pregnancy and obesity, laparoscopic, robotic or open surgery may still be indicated. In fact, robotic surgery has been increasingly used by urologists for different benign indications including chronic pyelonephritis, neglected ureteropelvic junction obstruction and renal tuberculosis. This article describes a case of robotic-assisted partial nephrectomy and nephrolithotomy for a large amount of small lithiasis in a non-functioning lower pole after failed stone clearance with a single use Boston ureteroscope owing to non-accessibility.

Keywords: urolithiasis, obstructive uropathy, poor functioning kidney, retrograde intrarenal surgery, robotic surgery, nephrolithotomy, partial nephrectomy
\end{abstract}

Volume II Issue 2 - 202 I

\author{
Jia Ying Isaac Tay,' Matthieu Gratton, ${ }^{2}$ Marc \\ Furrer, ${ }^{2}$ Jamie Kearsley ${ }^{2}$ \\ 'Department of General Surgery, The Royal Melbourne Hospital, \\ Australia \\ ${ }^{2}$ Department of Urology, The Royal Melbourne Hospital, \\ Australia
}

Correspondence: Jia Ying Isaac Tay, Department of General
Surgery, The Royal Melbourne Hospital,Australia,

Tel +6I 48I 309 012, Email jyisaac.tay@gmail.com

Received: March 01, 202I | Published: March 15, 2021

\section{Introduction}

In this age of advanced technology and strong emphasis on minimally invasive surgery, extracorporeal shockwave lithotripsy (ESWL), percutaneous nephrolithotomy (PCNL) retrograde intrarenal surgery (RIRS) have become standard treatments for renal stones depending on the stone location and size, and patient factors such as obesity, pregnancy, presence of aortic aneurysm, etc. However, laparoscopic, robotic or open surgery may still be indicated, when these calculi are not amenable or failed standard treatment. In conjunction, the use of robotic surgery has been expanding for various indications. It is useful for ureteral and large intrarenal stones, especially with underlying anatomic abnormalities. Procedures such as roboticassisted nephrolithotomy, pyelolithotomy and ureterolithotomy have been described by many large centres, with a stone free rate of 70$100 \% .{ }^{1}$ This case describes a robotic-assisted partial nephrectomy and nephrolithotomy for a large number of small stones in a nonfunctioning and dilated lower pole that were not accessible via RIRS.

\section{Case presentation}

A26-year-old Nepalese man was referred to the urologists for a large renal pelvic calculus found on an ultrasound done for hypertension workup. His blood pressures were up to $158 / 116 \mathrm{mmHg}$ and he was otherwise asymptomatic. A quadruple-phase computed tomography (CT) showed an obstructive $29 \mathrm{~mm}$ left renal pelvis calculus, Grade 4 hydronephrosis, and extensive small non-obstructing calculi in the left lower pole with cortical thinning (Figure 1A\&1B). The length of the left kidney was preserved at $11 \mathrm{~cm}$ due to the hydronephrosis, but the renal parenchyma was thin at $6 \mathrm{~mm}$, compared to the $10 \mathrm{~cm}-$ long right kidney with a parenchymal thickness of $11 \mathrm{~mm}$. He had preserved renal function with creatinine of $80 \mathrm{umol} / \mathrm{L}$. A 99mTc-MAG3 renogram showed reduced uptake and transit in the left lower kidney. There was no tracer drainage after Frusemide injection, with a half-life of 140min (Figure 2A). The differential left renal function was $40 \%$. A retrograde pyelogram performed revealed no ureteric abnormalities. Due to the multiloculated hydronephrotic appearance on $\mathrm{CT}$, renal tuberculosis was considered and ruled out with negative Quantiferon-gold testing. His other medical history includes asthma.

Due to the ongoing risk of chronic renal impairment and worsening of hypertension, the decision was made to treat the stone disease. RIRS was initially performed. A hard, impacted calculus was seen in the renal pelvis, and $75 \%$ clearance was achieved after two hours of laser lithotripsy with Holmium:YAG laser at 0.6joules and 20hertz. Another laser lithotripsy was repeated two months later using a Boston LithoVue ${ }^{\mathrm{TM}}$ Single-Use Digital Flexible Ureteroscope, which completely dusted the remaining pelvic stones. However, up to fifty more calculi were encountered in the lower pole, each measuring $3-6 \mathrm{~mm}$ in size. The infundibulopelvic angle was very acute, making it impossible to access the stones.

A 99mTc-DMSA was performed, showing irregular parenchymal thinning and scarring, and absent cortical uptake in the left lower kidney (Figure 2B). The left renal function was $44 \%$. A repeat noncontrast $\mathrm{CT}$ showed no more renal pelvis stones but still extensive calculi in the dilated lower pole (Figure 1C).

The options of management for the large stone burden was discussed in a multidisciplinary meeting. PCNL was unfavourable due to the thin renal cortices, which might result in loss of access during surgery, and nephrocutaneous fistula in the future. Furthermore, PCNL would not have corrected the issue of abnormal anatomy, and the dilated poor-functioning lower pole will lead to stone recurrence. Our patient was consented to have a robotic-assisted partial nephrectomy and nephrolithotomy.

A 4.8-Fr multilength JJ-stent and a 5-Fr ureteric catheter were inserted over sensor wires into the left ureter via cystoscopy. The patient was then placed in a right lateral position. We used a transperitoneal 4-arm approach with a 30-degree lens, fenestrated bipolar forceps, monopolar curved scissors, and prograsp forceps. After mobilising the descending colon and dissecting through Gerota's fascia, the grossly dilated and thinned left lower kidney was identified (Figure 3A). 


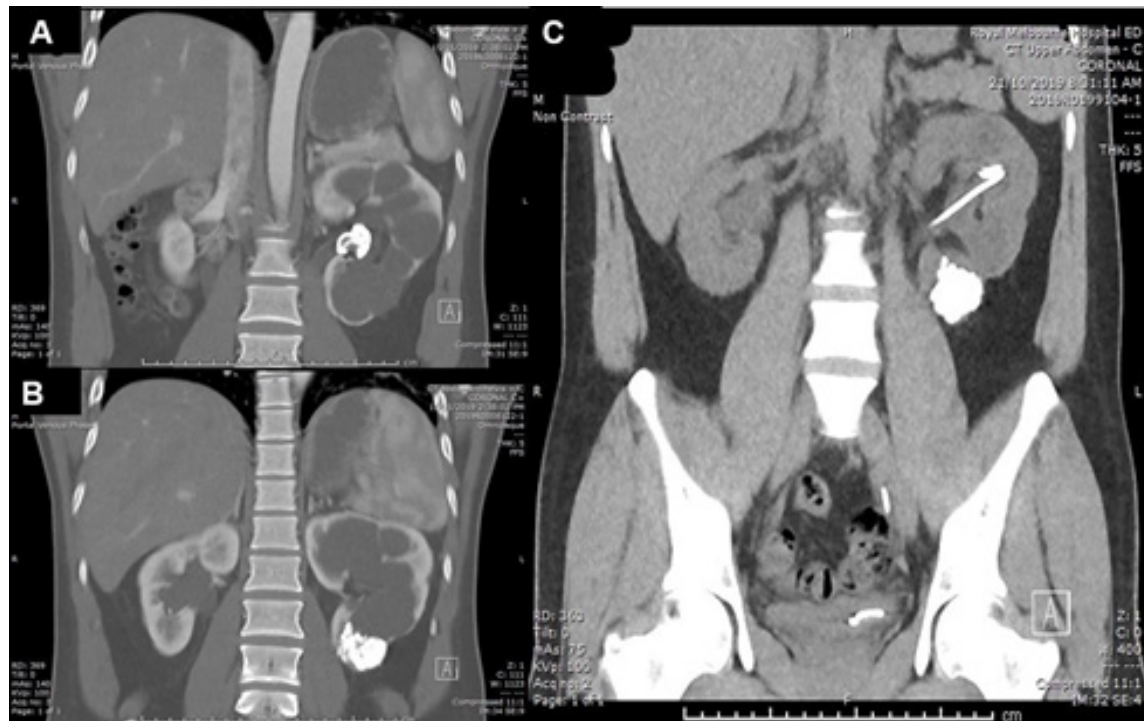

Figure I Pre and post RIRS CT images. (A) Initial scan shows left obstructing renal pelvic stone. (B) Initial scan also shows multiple small non-obstructing calculi in lower pole. (C) CT image post second RIRS demonstrate clearance of pelvic stone but residual calculi in lower pole.

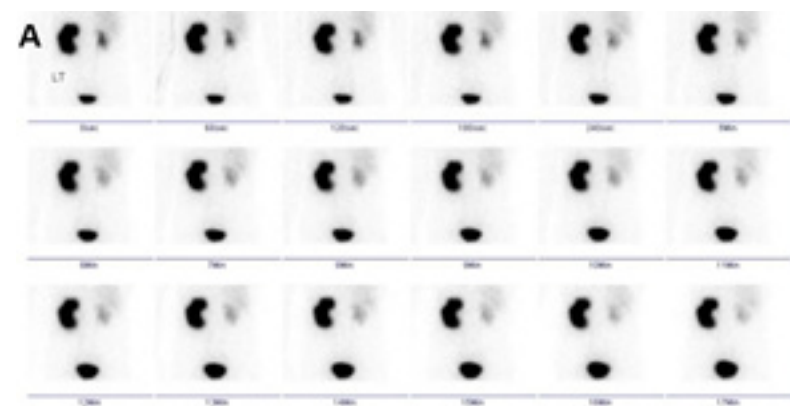

B
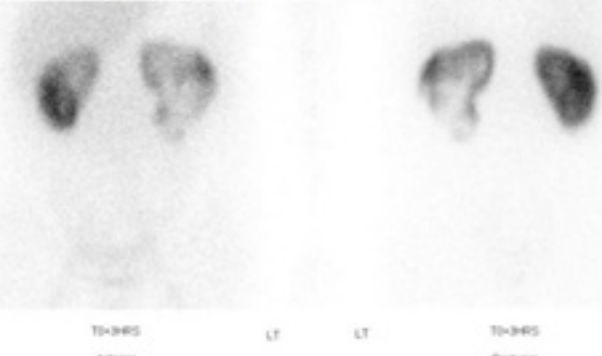

Figure 2 Nuclear renography assessments. (A) 99mTc-MAG3 renogram showing delayed and virtually no drainage of the radiotracer after Frusemide injection. (B) D99mTc-DMSA MSA showing irregular parenchymal thinning and scarring, and absent cortical uptake in the left lower kidney.
A $1 \mathrm{~cm}$ incision was made into the left lower calyx, and we encountered multiple small smooth stones inside, each around 3-6mm in size (Figure 3B). A 10mm laparoscopic suction tube was inserted into the calyx and used to aspirate the stones (Figure 3C). The incision was widened with curved scissors, which revealed more stones deep in the calyx. Up to fifty stones were extracted via suction as the partial nephrectomy was performed.

We excised and removed all four lower pole minor calyces (Figure 4A\&4B). Continuous V-Loc 3-0 sutures was used around the excision margin for haemostasis. The lower pole infundibulum was sutured closed and perinephric fat was re-adapted over the defect to reinforce and prevent leak from the renal pelvis. Methylene dye was injected through the ureteric catheter into the renal pelvis, which showed no leak, and the ureteric catheter was removed. Postoperative recovery was uneventful, and renal function was preserved. He was discharged 3 days post surgery. The JJ-stent was removed 4 weeks post-operatively.

Stone analysis revealed calcium oxalate composition. The patient was advised to adhere to a standardized stone prophylaxis. A postoperative non-contrast CT showed small volume of remnant stones at the lower pole infundibulum, measuring less than $6 \mathrm{~mm}$. His blood pressure was $129 / 80 \mathrm{mmHg}$. He was planned for a clinic follow-up and renal ultrasound in 6-months. Our patient will likely need a repeat RIRS in a year to treat the remaining stones.

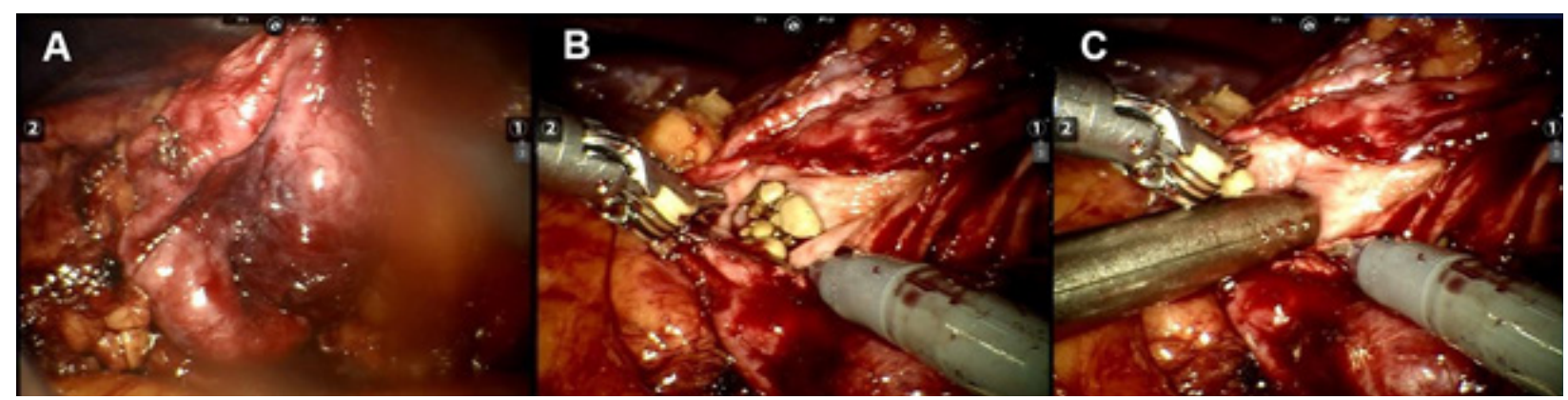

Figure 3 Nephrolithotomy being performed. (A) Dilated and thinned lower pole renal parenchyma encountered with a mass of calculi in-situ. (B) Numerous small calculi seen in the calyx. (C) The calculi were aspirated with a laparoscopic suction tube. 


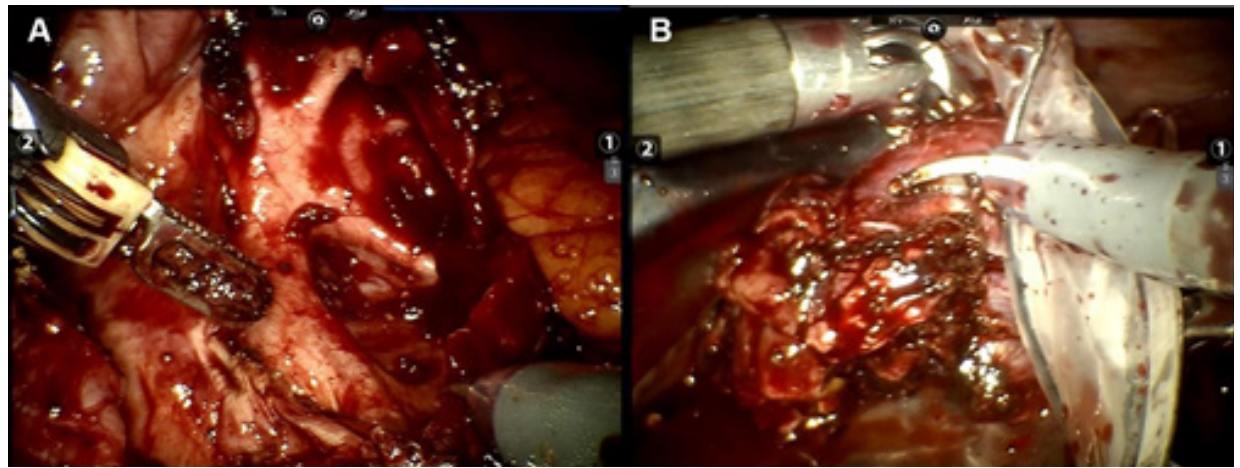

Figure 4 Partial nephrectomy being performed. (A) Excision of the grossly dilated minor calyces of the lower pole. (B) The excised left lower pole was removed via a specimen retrieval Endobag.

\section{Discussion}

There are well established guidelines for the renal calculi. Our patient's pelvic stone was $29 \mathrm{~mm}$, which provided the size indication for stone removal. For intrarenal stones larger than $20 \mathrm{~mm}, \mathrm{PCNL}$ is the recommended treatment, followed by RIRS or ESWL. ${ }^{2}$ However, PCNL was unsuitable in our case, as there are significant risks of fistula formation due to the thinned parenchyma. RIRS was performed twice which cleared the pelvic calculus, but not the lower pole stones.

The grossly dilated calyces created an infundibulopelvic angle of less than 45-degrees. Small infundibulopelvic angles has been described to predispose to calculus formation. ${ }^{3}$ This anatomical abnormality would also render both the access with a ureteroscope and passage of stones post ESWL near impossible. Furthermore, the large number of stones would make it a significantly difficult surgery and drastically lower clearance rates.

The significantly dilated lower calyces promote urinary stasis, which predispose to further stone formation, and consequently obstruction, infection or atrophy. This formed the main indication for partial nephrectomy, as PCNL would not have corrected that problem. The functional scans showed that the lower half was scarred with minimal function, and excising it will not reduce overall renal function. There is also evidence of better hypertension control after partial nephrectomy of atrophic kidney in children. ${ }^{4}$

Nephrectomy has been used for benign conditions such as chronic pyelonephritis, ureteropelvic junction obstruction and renal tuberculosis. ${ }^{5}$ Urolithiasis is an indication for nephrectomy when there are recurrent stones, non-functioning kidney or recurrent infections. Laparoscopic approach and partial nephrectomy were performed more frequently than open and radical ones. ${ }^{6}$ Large centres have described using laparoscopic pyelolithotomy, ureterolithotomy and nephrectomy for large long-standing renal and ureteral calculi.? Magdum et al. described a case of using laparoscopic ureterolithotomy for a $5 \mathrm{~cm}$ proximal ureteric stone. ${ }^{8}$ A study in India showed that robotic surgeries for lower ureteric stones had significantly shorter operative time, greater ease of surgery, with no conversion to open procedures required. ${ }^{9}$ Madi and Hemal reported 100\% stone free rate with robotic pyelolithotomy and nephrolithotomy, and $70 \%$ for anatrophic nephrolithotomy. ${ }^{1}$ Studies in the US and Turkey have reported concurrent robotic pyelolithotomy and pyeloplasty, with all patients stone-free 3 months post-op. ${ }^{10,11}$ Garisto et al. described a case of robotic pyelolithotomy and partial nephrectomy for a concurrent renal pelvis stone and an upper pole renal cell carcinoma. ${ }^{12}$

To our knowledge, this case study is the first reported roboticassisted partial nephrectomy and nephrolithotomy for multiples intrarenal calculus and atrophic lower pole. Robotic-assisted surgery offers the advantages of improved access and ergonomics, enhanced endo-wrist dexterity, precise hand-eye coordination and physiological tremor filtering. It is also beneficial for patients, with reduced morbidity, expedited recovery and decreased hospital stay. ${ }^{13}$

\section{Conclusion}

Stone surgery has well established treatment algorithms. However, the chosen treatment needs to be individualised based on the case particularities such as the stone burden, ease of renal pelvis access, etc, which have been highlighted in our case. Robotic surgery has its place in the treatment of urolithiasis as it can both treat difficult stones and correct anatomic abnormalities. In our case, roboticassisted partial nephrectomy and nephrolithotomy for multiple lower pole renal calculi and atrophic kidney provided great outcomes and recovery.

\section{Conflicts of interest}

The authors declare no conflict of interest.

\section{Acknowledgments}

None.

\section{Funding}

None.

\section{References}

1. Madi R, Hemal A. Robotic Pyelolithotomy, Extended Pyelolithotomy, Nephrolithotomy, and Anatrophic Nephrolithotomy. $J$ endourol. 2018;32(S1):S73-S81.

2. Türk C, Neisius A, Petř́ík A, et al. EAU Guidelines on Urolithiasis 2018 European Association of Urology Guidelines 2018 Edition. presented at the EAU Annual Congress Copenhagen 2018. Arnhem, The Netherlands: The European Association of Urology Guidelines Office. 2018.

3. Manikandan R, Gall Z, Gunendran T, Neilson D, Adeyoju A. Do Anatomic Factors Pose a Significant Risk in the Formation of Lower Pole Stones? Urology. 2007;69(4):620-624.

4. Baez-Trinidad LG, Lendvay TS, Broecker BH, et al. Efficacy of nephrectomy for the treatment of nephrogenic hypertension in a pediatric population. The Journal of urology. 2003;170(4 Pt 2):1655-1658.

5. Rafique M. Nephrectomy: indications, complications and mortality in 154 consecutive patients. J Pak Med Assoc. 2007;57(6):308-311.

6. Shrestha A, Chhettri P, Yadav BK, et al. Urinary Stone Disease and Preventable Nephrectomies. Journal of Nepal Health Research Council. 2019;17(2):238-241. 
7. Alyaev YG, Sirota ES, Bezrukov EA, et al. [3D-technologies in planning and navigation for laparoscopic interventions in patients with renal and ureteric stones]. Urologiia (Moscow, Russia : 1999). 2019(4):9-15.

8. Magdum PV, Nerli RB, Devaraju S, et al. Laparoscopic Ureterolithotomy for Giant Ureteric Calculus: A Case Report. Urology case reports. 2015;3(5):135-137.

9. Dogra PN, Regmi SK, Singh P, et al. Lower ureteral stones revisited: expanding the horizons of robotics. Urology. 2013;82(1):95-99.

10. Atug F, Castle EP, Burgess SV, et al. Concomitant management of renal calculi and pelvi-ureteric junction obstruction with robotic laparoscopic surgery. BJU international. 2005;96(9):1365-1368.
11. Mufarrij PW, Woods M, Shah OD, et al. Robotic dismembered pyeloplasty: a 6-year, multi-institutional experience. J urol. 2008;180(4):1391-1396.

12. Garisto JD, Dagenais J, Arora H, et al. Concurrent Robotic Pyelolithotomy and Partial Nephrectomy: Tips and Tricks. Urology. 2018;118:243.

13. McGuinness LA, Prasad Rai B. Robotics in urology. Ann R Coll Surg Engl. 2018;100(6sup):38-44. 